\title{
An Effective Heuristics Approach for Performance Enhancement of MANET
}

\author{
Khoshal Rahman Rahmani, Md Sohel Rana, Md Alamin Hossan, \\ And Wali Mohammad Wadeed
}

\begin{abstract}
Mobile Ad-hoc Networks (MANET) are widely adopted in almost all research fields due to their significant advantages like minimal energy consumption and compact size. In MANET major challenges are optimal coverage, a lifetime of nodes, and throughput. This paper proposed a hybrid ant colony-flower pollination (HAC - FP) algorithm for throughput maximization and minimal energy consumption in a sensor network. To enhance the performance of the MANET network this research adopts the meta-heuristic technique. The metaheuristic approach utilized for the MANET network is the ant colony and flower pollination algorithm. The planned HAC-FP makes use of neighborhood distance to determine the best position for each node. With hypersphere localization, the Levy struggle in flower pollination is used for optimal energy location. The placement of sensor nodes is identified in the first step using a hypersphere. Sensor network node energy consumption is lowered based on neighborhood distance. The results showed that the suggested HAC-FP algorithm, rather than existing methodologies, enhances the MANET network's coverage area. Further proposed HAC-FP algorithm minimizes the energy consumption level of the MANET than the conventional genetic approach.
\end{abstract}

Keywords - Mobile Ad-hoc Network, Neighbourhood Distance, Levy Flight, Flower Pollination, Ant Colony.

\section{INTRODUCTION}

Mobile Ad-hoc Network (MANET) relies on the group of ad-hoc networks integrated with sensing devices for reducing cost and minimizing the amount of energy consumed through wireless links that are interconnected. The significant advantage of MANET is resource constraint is minimal and compact sensor node [1]. The infrastructure of MANET is similar to characteristics of Wireless sensor network (WSN), in terms of self-organized, distribution, and multi-hop scenario except for the fact infrastructure mobility. The comparative analysis of WSN and MANET varies in the different scenarios in terms of bandwidth, reduced cost, enhancement in power constraints level, and data redundancy [2], [3]. In the case of, industrial application MANET has been employed for the monitoring process. At this point, information is exchanged continuously for data confidential communication, security, reliability, and network infrastructure level [4], [5]. Wireless communication those are having nodes with non-intended scenarios that have

Submitted on December 18, 2021

Published on January 17, 2022.

Koshal Rahman Rahmani, Afghanistan.

(e-mail: khoshalrahman.rahmani@gmail.com)

Md Sohel Rana, NUIST, China, Bangladesh.

(e-mail: sohelrana117373@gmail.com) eavesdroppers which lie in the communication range for transmission overhead and extraction of private information [5]. To provide MANET communication in a secure manner cryptography technique has been utilized for the hierarchical layer of the communication protocol for offering data confidentiality [6]. To offer effective performance in comparison with WSN, the MANET network has been evolved but still, it requires certain changes rather than WSN those are: 1. Aggregated data are collected in cluster head leads to data redundancy or data disparate and consumption of energy also higher while sending those redundant data; 2 . The network routing strategy is difficult to handle hence minimal information needs to be adopted in routine during the establishment of a path using cluster head which significantly improves the network scalability; 3 . The link between $\mathrm{CHs}$ and sensor nodes is established using a communication link hence higher bandwidth for communication is conserved with no redundant data exchange. Overall MANET network requires reduced energy consumption level and improved throughput for the effective performance of MANET.

To withstand those requirements of MANET researchers developed a metaheuristic approach for sensor network identification and localization. Using effective communication capabilities with search space optimal solution metaheuristics provide an excellent solution. Based on the trial and error performance heuristics algorithm offers a significant solution for providing an effective solution using adaption and learning. Even though it does not offer an excellent solution instead it provides an acceptable solution over a certain period. A few significant benefits of this heuristic approach are it exhibits faster computing time in comparison with other methods also it is a minimal price in comparison with other computing techniques [7], [8]. Also, the heuristics approach provides significant equality between processing time and results but still in some instances heuristics approach fails for the identification of an optimal solution. At present, meta-heuristics protocols are compatible with resolving issues related to optimization. Within the acceptable time, constraints scenario using the metaheuristics approach optima solution is achieved with increased energy and network efficiency. This leads to an effective impact on the system's time [9]. In MANET network optimization algorithms such as Ant-Colony, Particle Swarm Optimization

Md Alamin Hossan, Daffodil Int University, Bangladesh.

(e-mail: alamin311293@gmail.com)

Wali Mohammad Wadeed, Afghanistan.

(e-mail: wadeed.walid@gmail.com) 
(PSO), Krill-Heard, Flower Pollination algorithm exhibits significant performance over a network.

This paper proposed a HAC-FP to improve throughput with reduced energy utilization. The proposed algorithm combines Ant colony and Flower pollination algorithm are combined in this research based on neighborhood distance approximation and levy fight criteria. The suggested algorithm combines several techniques. This study combines an ant colony and a flower pollination algorithm based on neighborhood distance approximation and levy conflict conditions. The proposed HAC-FP makes use of neighborhood distance to determine the best position for sensor nodes. With hypersphere localization, the Levy struggle in flower pollination is used to find the best energy position. The first phase, which is based on the hypersphere, is used to locate sensor nodes. Sensor network node energy consumption is lowered based on neighborhood distance. Matlab Simulator was used to test the performance of HACFP, and the findings showed that the suggested method increased throughput while consuming less energy.

The following is how the paper is structured: In this section, I present an overview of MANET and the metaheuristic algorithm. In part II, relevant efforts for throughput maximization and energy consumption are presented. Section III organized the paper, and Section IV offered a full description of the problem formulation for optimal sensor node placement. The proposed HAC-FP algorithm with neighborhood and hypersphere criteria was discussed in section V. The simulation findings were provided in Section VI, and the research's conclusion was described in Section VII.

\section{RELATED WORKS}

Researchers have conducted numerous methods for improving the throughput of the network with the utilization of various power allocation strategies. Benaya et al. [20], developed an allocation strategy for optimal flow of power using a Genetic algorithm. This research focused on the interference of channel and throughput of the network. Through analysis, this research identified that with the utilization of amplification factor throughput has been maximized with consideration of power constraints through effective sharing of spectrum. Another researcher, Rosas et al. [21] adopted a genetic algorithm technique for improving power allocation strategy with consideration of every subcarrier with maximal throughput observation. Panaitopol et al. [22] evaluated the sensing characteristics with consideration of symbol length and cyclic prefix of the network through a combination of cyclo-stationary properties of the network to improve the throughput of the network. Even though Inzucchi et al. [23] applied Widely Linear Estimation (WLE) for symbol error rate (SER) analysis.

As per researcher, Zewail et al. [24], the network is evaluated using access point and throughput measurement of the network for interference cancellation with consideration of power level optimal value for improving data rate. Similarly, another researcher Lin and Siohan [25] investigated the performance of networks along with frequency-domain characteristics of networks with consideration of filter sparsity through characteristics of orthogonality.

\section{Proposed Methodology}

In a wider range of application data collision and redundancy is major concern for the transmission of data hence proposing a hybrid metaheuristic algorithm. In wireless communication, users are evaluated in circles unequal which utilize circumstance with an application of wider spectrum [10]-[13]. To resolve, these parts need to be placed in a rigid form. To evaluate the framed problem designed wireless communication adopts

Consider a square spectrum which is denoted as $\mathrm{S}$ and its side length are defined as $\mathrm{L}\left(\mathrm{L} \geq 2 \mathrm{r}_{\mathrm{n}}\right)$ and corresponding circles are stated as $\mathrm{C}_{1}, \mathrm{C}_{2} \ldots . ., \mathrm{C}_{\mathrm{n}}$ also $(n \in N)$ for those corresponding circles in the node network, the circle radius in fixed $\mathrm{r}_{1}, \mathrm{r}_{2}, \ldots$, and its condition is denoted as $r_{1} \leq r_{2} \ldots . . r_{n}$. In 2-dimension coordination, the system considers spectrum sides are parallel, the corresponding sides of the coordinate are $\mathrm{C}_{\mathrm{i}}(\mathrm{i} €[1, \mathrm{n}])$ be $\left(x_{i}, y_{i}\right)$. Thus, the main objective of this scenario is a reduction of possible value meantime of locating this circle without any collision in a MANET network. The problem solution is denoted as $(L, X)$ in which configuration of the network is stated as $\left(\mathrm{x}_{1}, \mathrm{y}_{1 \ldots .}, \mathrm{x}_{\mathrm{i}}, \mathrm{y}_{\mathrm{i}}, \ldots \ldots \ldots, \mathrm{x}_{\mathrm{n}}, \mathrm{y}_{\mathrm{n}}\right)$. Also, the formulated objective function is presented as follows:

Minimize L, s.t

$$
\begin{gathered}
\left|x_{i}\right|+r_{i} \leq \frac{L}{2}, \forall 1 \leq i \leq n \\
\left|y_{i}\right|+r_{i} \leq \frac{L}{2}, \forall 1 \leq i \leq n \\
\sqrt{\left(x_{i}-x_{j}\right)^{2}+\left(y_{i}-y_{j}\right)^{2}} \geq r_{i}+r_{j}, \forall 1 \leq i<j \leq n
\end{gathered}
$$

The above-mentioned formula (1) and (2) define the MANET network collision. In this, both equations (1) and (2) rely on collision lies within the circle located in MANET. The above equation (3) minimizes collision lies in the MANET network. The analysis of equation (3) is satisfied then it is feasible else it is not. To minimize the process of optimization which is involved in process of intermediate through overlaps process this is utilized for function evaluation. The evaluation function is combined with other factors such as $\left\langle\mathrm{L}_{\mathrm{CLB}}, \mathrm{E}(\mathrm{X})\right\rangle$ with consideration of unequal circles as $\langle\mathrm{R}, \mathrm{E}(\mathrm{X})\rangle$.

The potential energy function of the network is presented as $E(X)$ which measures infeasibility that occurred due to collision which is defined as below:

$$
E(X)=\sum_{k=1}^{n} \frac{1}{2}\left(O_{v k}^{2}+O_{h k}^{2}\right)+\sum_{i=1}^{n-l} \sum_{j=i+1}^{n} \frac{1}{2} O_{i j}^{2}
$$

In the below equation collision in a network is denoted as $O$ in other hands, the collision between circle kalong with sides in vertical form is:

$$
O_{v k}=\operatorname{Max}\left\{\left|x_{k}\right|+r_{k}-\frac{L}{2}, 0\right\}
$$


In case of a horizontal side collision insides of a circle is represented as $O_{h k}$ also it defines overlap of circle k:

$$
O_{h k}=\operatorname{Max}\left\{\left|y_{k}\right|+r_{k}-\frac{L}{2}, 0\right\}
$$

$O_{i j}$ is the overlap between circle $i$ and circle $j$ :

$$
O_{i j}=\operatorname{Max}\left\{r_{i}+r_{j}-\sqrt{\left(x_{i}-x_{j}\right)^{2}+\left(y_{i}-y_{j}\right)^{2}}, 0\right\}
$$

The above equation (6) and (7) stated the limit lies in the range of $L \geq 2 r_{n}$, those all do not overlap with consideration of vertical sides. The optimization approach evaluates the function $\left\langle L_{C L B}, E(X)\right\rangle$ with an ingredient of key function $L_{C L B}$. The feasible solution objective function length $L$, for evaluation of function. In this scenario, solution feasibility is difficult for analysis for the same length for identification of a significant solution. To resolve those difficulties lies with the system the compact length of the system is denoted as $\left(L_{C}\right)$ for a compact and feasible solution.

\section{A. Proposed Hybrid Ant Colony - Flower Pollination (Hac-Fp)}

The proposed approach aims to enhance the throughput of the MANET network through the reduction of energy in the system. Hence the proposed approach utilizes optimization algorithm Ant colony and Flower pollination through consideration of levy fight approximation and neighborhood distance calculation.

Input: An initial solution input is $\mathrm{S}$; where basic neighborhood (BN); the candidates' path length search is (SPLList); supplementary neighborhoods candidate is (SNList).

Output: Identification of best solution through search.

1 repeat.

$2 S \leftarrow$ Best Improvement (S, BN); \% Descent Part: uses Best Improvement search to repeatedly achieve percent improvements until reaching a local minimum.

$3 S P L \leftarrow S P L$ Lis $t[0]$; \% SPL List's first value is the length of the starting search path.

$4 S N \leftarrow S N$ List [0]; \% initial supplementary neighborhood as the first neighborhood in SN List;.

5 repeat \% Breakthrough Part: To gain a further improvement, Tabu search and Variable Neighborhood percent Descent are used alternately.

$6 S \leftarrow T S$ Oscillation $(S, B N, S P L) ; \%$.

7 if no improvement has occurred

8 SŁOne Iteration $V N D(S, S N) ; \%$.

9 end if

10 SPL $\leftarrow$ Schedule Search path length (SPL, SPL List); \% section $4.5 \%$ Choose the next value in SPL List as current search path length.

11 SN $\leftarrow$ Schedule Neighborhood (SN, SN List); \% section $4.5 \%$ Choose the next neighborhood in SNList as current supplementary neighborhood.

12 until an improvement has occurred or $(S P L<0)$.

13 until $S P L<0$ and $S N<0 ; \%$ Brek through part has failed to provide a further improvement.
The developed hybrid heuristic approach with ant colonyflower pollination algorithm integrates procedure for preceding together. In every iteration proposed HAC-FP employs for Best Improvement of present best solution $\mathrm{S}$ with the efficient neighborhood. The nearby nodes in the MANET are evaluated using the below procedure:

B. Algorithm of Proposed HAC-FP

\begin{tabular}{|l|}
\multicolumn{1}{|c|}{ Algorithm of Proposed HAC - FP } \\
\hline Input: Number of ant individual (sensor Node) \\
Output: Mobile Ad-hoc Network Neighbourhood Distance \\
Initialization (Population, Sensor nodes and Space) \\
Repeat (iterationcount 1000 for threshold didentification) \\
For $\mathrm{i}=0$ to $\mathrm{k}-1$ do \\
Initialize the netser $=\varphi$ \\
Based on constraint solution step by step network start for sinknode detection \\
Evaluate acceptance fuction \\
End for \\
If (Trigger current iteration operation acceptance) \\
Evaluate acceptance finction \\
Calculate comvergence of sensor node \\
End if \\
If \\
Inplement infuence finction to calculate population space \\
End if For iteration do \\
Calculate increment of pheromone; \\
End for \\
For each point in workspace do \\
Update pheromone \\
End for \\
End
\end{tabular}

\section{System model}

Consider aggregator node central unit distance of $\mathrm{D}>0$ with an assumption of relay node existence between relay nodes which employed for decode and strategy of forwarding mechanism. In the straight-line relay, nodes are deployed that are utilized for aggregation and central unit evaluation with consideration of distance $\mathrm{d}>0$ among the nodes. In the MANET hop lies in the network are computed using $h=D / d$ specifically for the scattered nodes that are spread randomly for the existence of communication between central unit and aggregator. Based on the throughput and link for multi-hop evaluation is performed [9]:

$$
T=\frac{\log (1+\beta)}{h}\left(P_{\text {suc }}\right)^{h}
$$

At the receiver side, successful reception of the message is stated as $P_{\text {suc }}$ which has SIR minimal range of $\beta>0$. Through the consideration of assumption Gaussian codes and decoding, the rule is utilized for the calculation of interference as noise in a network [8]. The designed MANET efficiency of the spectrum is defined using $\log (1+\beta)$ which has been measured using bits $/ \mathrm{s} / \mathrm{Hz}$, for a single-hop range of SIR $>\beta$.

In the constructed MANET network hoping in long-stay is defined by $h=1$ with throughput range of $\log (1+\rho)$ Psuc [14]. The network components lie in consideration of two channels such as fading and distance-dependent systems. Receiver node power is examined using $\mathrm{g}_{\mathrm{ir}}-\alpha_{\mathrm{i}}$, and the reference distance of $i^{\text {th }}$ node and its $g_{i}$ is channel gain with 
consideration of path-loss exponent stated as $\alpha>2$ [5]. The transmitter time slots are [15] evaluated using location and channel gain. The SIR is calculated using formula (9):

$$
\begin{gathered}
S I R=\frac{g_{0} d^{-\alpha}}{\sum_{i \in \Phi_{\text {int }}} g_{i} r_{i}^{-\alpha}} \\
f_{R_{1}}(r)=\lambda_{\text {eav }} 2 \Pi r e^{-\lambda_{\text {eav }} \Pi r^{2}}
\end{gathered}
$$

$\lambda_{\text {eav }}$ defines the process of Poisson point in the network [7].

The MANET network outage probability is evaluated using below equations (11) and (12):

$$
\begin{gathered}
P_{\text {out:eav }}=E_{r}\left[1-e^{-\lambda_{\text {int }} k \prod r^{2}\left(\beta_{\text {eav }}\right)^{2 / \alpha}}\right] \\
=\frac{\lambda_{\text {int }} k\left(\beta_{\text {eav }}\right)^{2 / \alpha}}{\lambda_{\text {int }} k\left(\beta_{\text {eav }}\right)^{2 / \alpha}+\lambda_{\text {eav }}}
\end{gathered}
$$

where $\operatorname{Er}[\cdot]$ is represented as the value of expected distance $r$. Hop length is defined as $d$ and threshold value of SIR of $\beta$ for multi-hop optimization to achieve throughput. The distance has been calculated using the below formula (13)

$$
\begin{aligned}
T & =\frac{d \log (1+\beta)}{D}\left(e^{-\lambda_{\text {int }} k \prod d^{2} \beta^{2} / \alpha}\right)^{D / d} \\
& =\left(\frac{\lambda_{\text {int }} k\left(\beta_{\text {eav }}\right)^{2 / \alpha}}{\lambda_{\text {int }} k\left(\beta_{\text {eav }}\right)^{2 / \alpha}+\lambda_{\text {eav }}}\right)^{D / d}
\end{aligned}
$$

Equation (15) involves the probability constraint over eavesdropper links for the multi-hop link outage probability of the network.

\section{Formation of Ch with Hac-Fp}

This section presented a level-based approach for clustering where $\mathrm{CHs}$ are formulated through an energyaware system using the consideration of threshold function. The nodes which have the highest probability will have the chance of cluster head. Every node generates a random number and tries to become $\mathrm{CH}$ in case of a value that is minimal then the random value is less than the threshold this will become $\mathrm{CH}$. This can be evaluated using formula (15):

$$
T(i)=\frac{P_{o p t}}{1-p_{o p t}\left(r \cdot \bmod \left(\frac{1}{P_{o p t}}\right)\right)} \times \frac{E_{i}(r)}{E_{a v g}(r)}
$$

It has been considered that network nodes have the energy level of $E_{i}(r)>0$ n which $\mathrm{r}$ is denoted present round lifetime of the network, in which energy level of a present node is $E_{i}(r)$ energy remains in the node are represented as $E_{\text {avg }}$ and current node $\mathrm{I}$ is defined as $E_{\text {avg }}=\frac{\sum E_{i}(r)}{N}$, the total node in the MANET is stated as N.

\section{E. Network Energy Model}

For analysis, this research involved the deployment of the "N" node randomly for the network field of M/N. Also, every node in the nodes is stationary, and each node is assigned a unique identification number. In deployed node information send or received utilizes few energies with respect to a model of channel propagation with free space for transmission in one-hop or direct and channel of multipath fading mechanism [16]. In equation (16) presented about multipath mechanism mathematical model in the MANET network:

$$
E_{n} T_{r x}(L, D)=\left\{\begin{array}{l}
L E_{n(\text { elec })}+L \varepsilon_{\text {fres }} D^{2}, D<D_{0} \\
E_{n(\text { elec })}+L \varepsilon_{m_{\text {pat }}} D^{4}, D \geq D_{0}
\end{array}\right.
$$

In the above equation, data packet size is stated as $\mathrm{L}$, free space energy loss is denoted as $\varepsilon_{\text {fres }}$, and multipath energy loss is represented as $\varepsilon_{m_{p a t}}$. Threshold distance of $D_{0}$ is defined as $\varepsilon_{\text {fres }}$ or $\varepsilon_{m_{\text {pat }}}$. In which, a threshold value is expressed using below equation (17):

$$
D_{0}=\sqrt{\frac{\varepsilon_{\text {fres }}}{\varepsilon_{m_{p a t}}}}
$$

\section{F. Node Deployment Based on Proposed Han-Fp}

To identify an optimal solution for resolving deployment of node it is necessary to derive the solution to withstand coverage and connectivity among them for the satisfaction of optimal solution. The derivation of deployment of node effectively performed in coverage and connectivity of system through utilization of ant colony algorithm which is used for approximation but not used for deriving an optimal solution. To seek an optimal solution any system obtains a solution to derive constraints for satisfaction and derive constrained solution for output [17]. In the optimization approach space of population is evaluated spontaneously in which few spaces are accepted with consideration of population of the individual with the influence and evolutionary process with respect to ant space [18]. The development of the evolutionary mechanism does not receive a stable MANET mechanism, but it enhances the network efficiency and stability using ant colony optimization. In this number of nodes deployed are evaluated equally with consideration of ant individual. In which, ant individual fitness value is evaluated based on the deployed sensor with MANET coverage and connectivity for obtaining individual with minimal fitness function. 

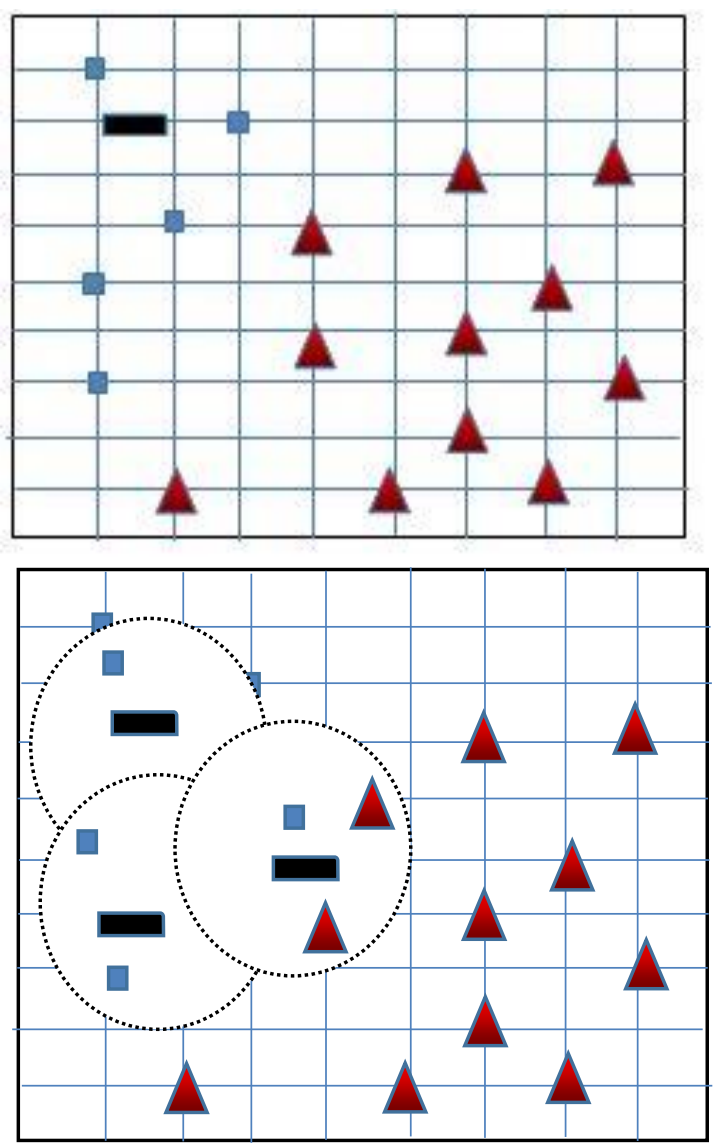

Fig.1. Hyper Sphere Sensor Optimization

Individual ant in the system provides pheromone in its part, but this has been evaporated with respect to environmental change and time-lapse in the system. In equation (18) formula for updating node are presented combined with the flower pollination approach.

$$
\tau_{i j}=\rho \tau_{i j}+\Delta \tau_{i j}^{k}
$$

where $\mathrm{q}$ represents the coefficient of pheromone which is evaporated and $(1-\rho)$ provides the pollination that occurred in the framed network. The objective of using the evaporation process through the grid is by means of iteration and which minimizes the effectiveness of the system through the total number of ants and flowers. The coefficient involved in natural selection or evolution is evaporation. The enhanced pheromone in pointgrid is $\tau_{i j}^{k}$

$$
\Delta \tau_{i j}^{k}=\frac{Q}{\text { SensorUsed }}
$$

The integration of ant colony and flower pollination is involved in the implementation of accumulation of pheromone faster for higher optimal locations [19]. Also, pheromone and pollination mechanisms involved the accumulation of faster convergence accumulation by means of suboptimal solution [17]. To overcome the accumulation of pheromone in unlimited value limited conditions are applied which restrict the higher pheromone concentration point of ants with minimal ants. The level of pollination is presented as follows:

$$
\tau_{i j}=\left\{\begin{array}{l}
\tau_{-} \text {MAX, pher }>\text { pher_MAX } \\
\tau_{i j}, \text { pher_MIN }<\text { pher }<\text { pher_MAX } \\
\tau_{-} \text {MIN, pher }<\text { pher_MIN }
\end{array}\right.
$$

where $\tau_{i j}$ represent the grid point pheromone concentration, pher_x0005_MIN and pher_x0005_MAX denote the pheromone minimal and maximal value.

\section{EXPERIMENTAL ANALYSIS}

This section presented the experimental setup evolved for the analysis and design of the proposed approach for collision minimization in the MANET network.

\section{A. Experimental Settings}

For the simulation of the proposed algorithm MATLAB simulation software is used for evaluation. The simulated results are comparatively examined with other existing genetic algorithms in which MANET is designed with 50 nodes with an overall coverage area of $100 \mathrm{~m}^{2}$. Also, it has an initial energy level of 0.1 where the optimal placement of nodes is placed in 100 iterations. The designed network parameters are tabulated in below Table I.

\begin{tabular}{cc}
\multicolumn{2}{c}{ TABLE I: DESIGNED NETWORK PARAMETERS } \\
\hline Parameter & Value \\
\hline Area (x, y) & 100,100 \\
Base station (x, y) & 50,50 \\
Nodes (n) & 100 \\
Probability (p) & 0.1 \\
Receiver_energy & $50 \times 10^{-9}$ \\
Multipath (amplifier) & $0.0013 \times 10^{-13}$ \\
Effective rate of data aggregation & $5 \times 10^{-9}$ \\
Maximal Network Lifetime & 2500 \\
Packet size of data & 4000 \\
Network energy in initial state & 0.1 \\
Transmiter_energy & $50 \times 10^{-9}$ \\
\hline
\end{tabular}

Residual energy is seen as a critical requirement for nodes in the MANET network, as it determines the network longevity. The residual energy level, which has been compared using ant-colony and genetic algorithms, must be examined to determine the success of the suggested hybrid strategy. The deployment of different nodes in the network has been analyzed for evaluation purposes. The energy level of nodes in the network is shown in Table II for various node counts.

TABLE II: ENERGY EVALUATION OF PROPOSED APPROACH

\begin{tabular}{cccccccc}
\hline \multirow{2}{*}{ Algorithm } & \multicolumn{7}{c}{ Nodes } \\
\cline { 2 - 8 } & 100 & 150 & 200 & 250 & 300 & 350 & 400 \\
\hline Genetic & $0.445 \pm 0$. & $0.274 \pm 0$. & $0.4742 \pm 0$ & $0.3422 \pm 0$ & $0.2854 \pm 0$. & $0.357 \pm 0$. & $0.4475 \pm 0$ \\
algorithm & 1945 & 2356 & .2457 & .2045 & 28656 & 2174 & .23575 \\
Artificial bee & $0.356 \pm 0$. & $0.264 \pm 0$. & $0.4584 \pm 0$ & $0.3554 \pm 0$ & $0.2545 \pm 0$. & $0.3876 \pm$ & $0.4785 \pm 0$ \\
colony & 20455 & 1954 & .1465 & .13655 & 1855 & 0.22547 & .1754 \\
Proposed & $0.5545 \pm 0$ & $0.3452 \pm 0$ & $0.4542 \pm 0$ & $0.4785 \pm 0$ & $0.2475 \pm 0$. & $0.3542 \pm$ & $0.4521 \pm$ \\
& .15565 & .28476 & .16651 & .1956 & 1545 & 0.1955 & 0.1655 \\
\hline
\end{tabular}


TABLE III: THROUGHPUT ANALYSIS

\begin{tabular}{cccccccc}
\hline \multirow{2}{*}{ Algorithm } & \multicolumn{7}{c}{ Nodes } \\
\cline { 2 - 8 } & 100 & 150 & 200 & 250 & 300 & 350 & 400 \\
\hline Genetic & $8.153 \pm$ & $11.35582 \pm$ & $16.2671 \pm$ & $21.9552 \pm$ & 24.5423 & $27.3175 \pm$ & 32.5206 \\
algorithm & 2.4475 & 2.9565 & 2.856 & 3.145 & \pm 3.445 & 3.8445 & \pm 2.9455 \\
Artificial bee & $9.245 \pm$ & $10.454 \pm$ & $17.645 \pm$ & $22.74852 \pm$ & 23.34785 & 28.27872 & $33.678 \pm$ \\
colony & 2.355 & 1.9455 & 3.056 & 2.845 & \pm 3.5546 & \pm 2.945 & 3.2445 \\
& $10.4745 \pm$ & $12.674 \pm$ & $18.57582 \pm$ & $23.9751 \pm$ & $23.6475 \pm$ & $29.1856 \pm$ & 34.7555 \\
Proposed & 3.854 & 2.454 & 2.6465 & 2.945 & 3.445 & 2.34552 & \pm 2.7554 \\
\hline
\end{tabular}

The above Table II illustrate node number in comparison with the genetic algorithm with the proposed approach. Through analysis, it is observed that the proposed approach exhibits residual energy significantly. Due to random deployment fluctuation is observed in the network. The analysis of throughput is measured are presented in Table III. In comparison with the existing technique proposed approach exhibits significant throughput of $2.7897 \%$ rather than other approaches. Hence through analysis, it is concluded that it proposed results offer significant performance rather than the existing approach.

Generally, MANET differs in the density of the network in terms of dense and sparse. The packet size utilized for analysis is 1024 bits with an evaporation rate of 0.25 . In the case of deployment of nodes, iteration counts considered are 10, 100, and 1000. Simulation node deployment is illustrated in Fig. 1 and Fig.2. In another figure iteration process of nodes is presented.

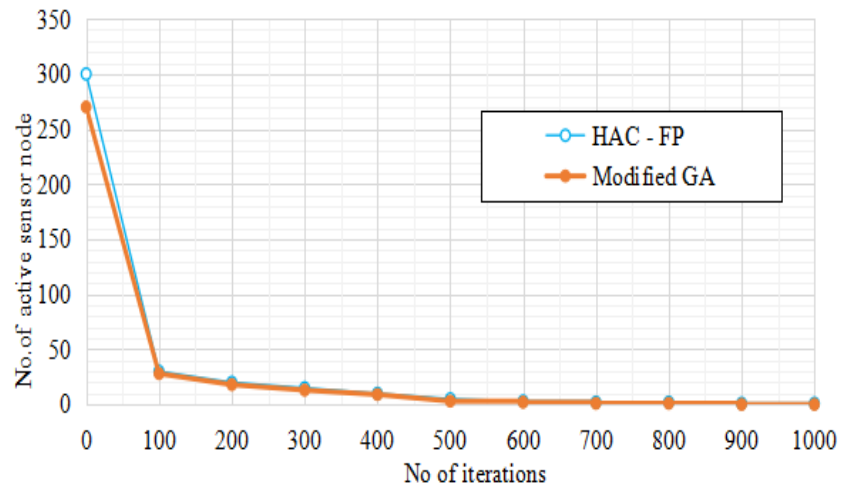

Fig.2. Active Sensor Node Count before Neighborhood Distance.

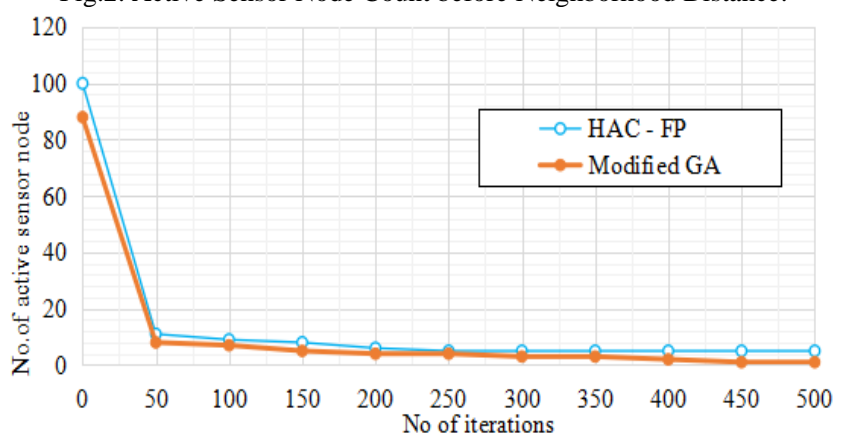

Fig.3. Active Sensor Node Count after Neighborhood Distance

Calculation The below figure illustrates the source node and sink node of deployed nodes in the network with the selection of cluster head selection.

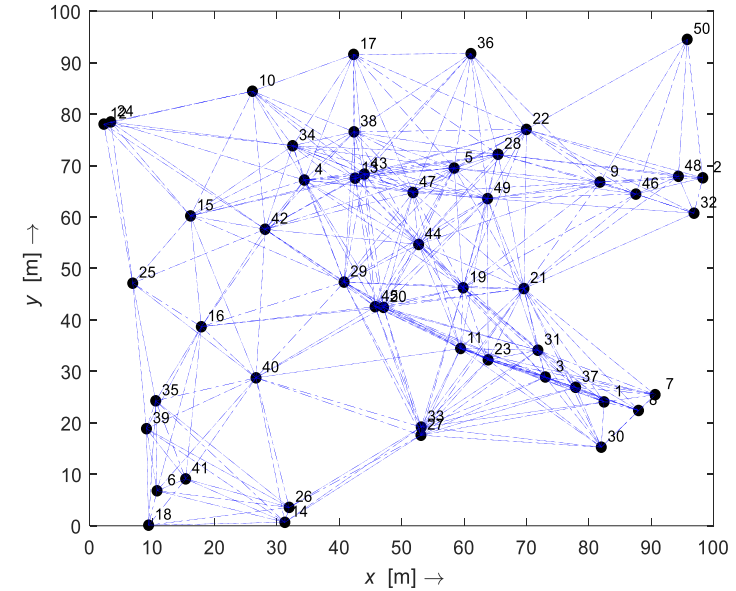

Fig.4. Initial Sensor Location at iteration 1 for neighborhood distance calculation.

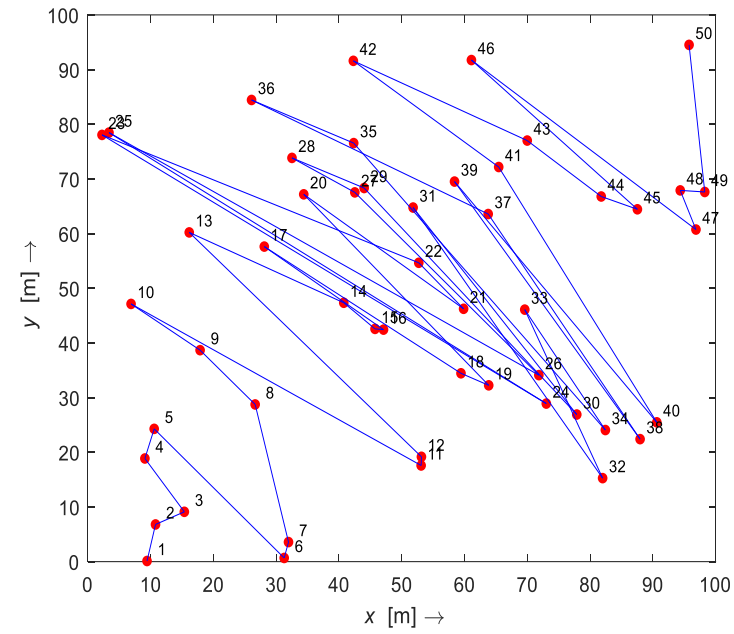

Fig.5. Sensor Node Location at iteration 100 for neighborhood distance calculation with hyper Sphere.

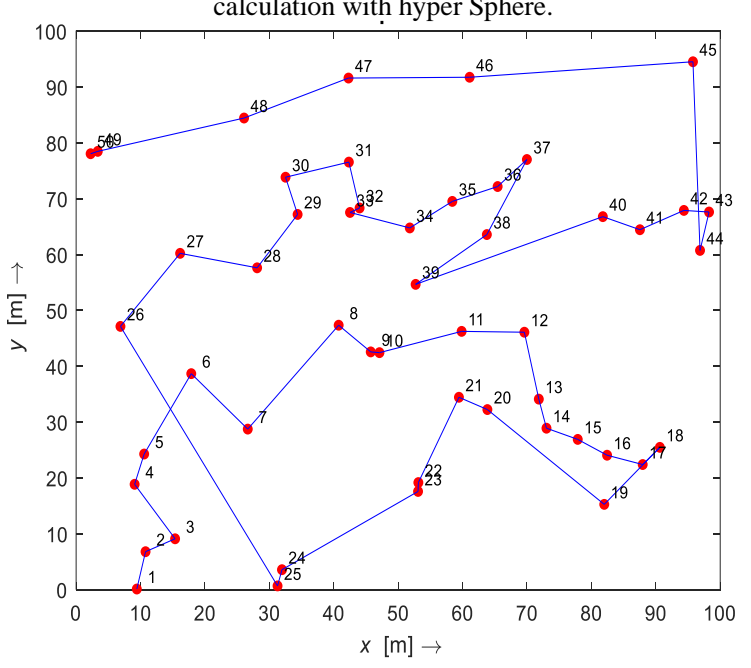

Fig.6. Sensor Location at iteration 1 for HAC- FP. 


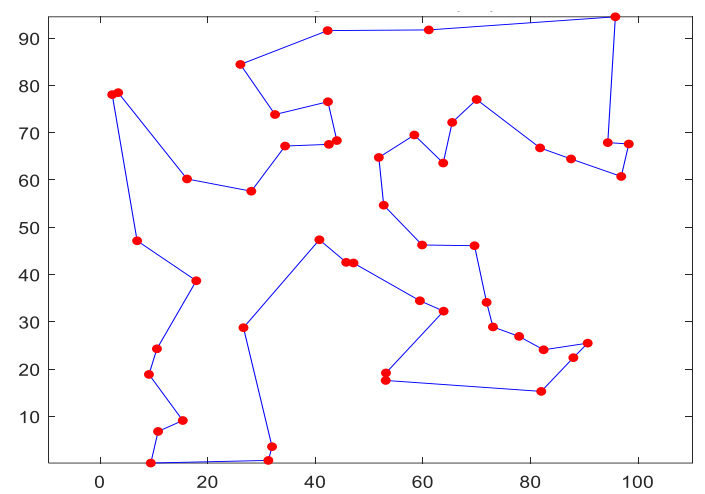

Fig.7. Sensor Location at iteration 10 for HAC-FP.

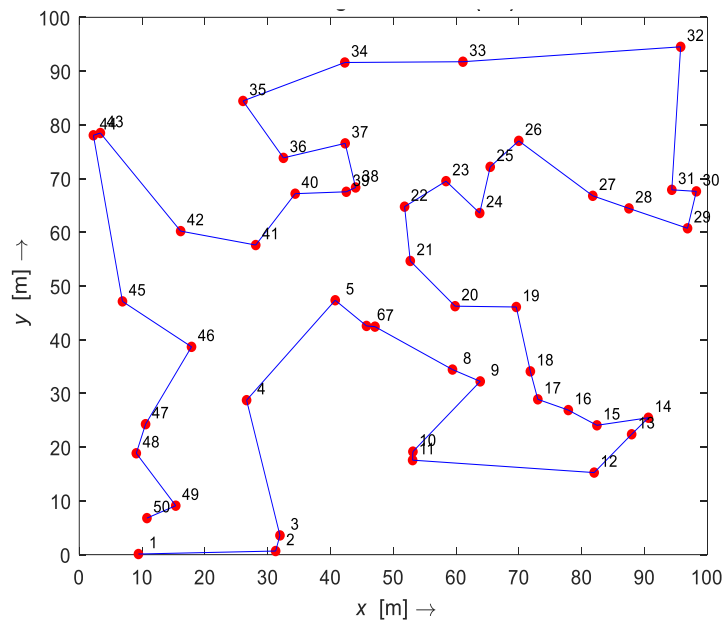

Fig.8. Sensor Location at iteration 100 for HAC-FP.

The above Fig.5-9 shows the node deployment for a total of 100 iterations in the system. Further Fig.5 demonstrates MANET network initial network condition while in figure 6 and 7 neighborhood calculation is presented. The above Fig. 8 and 9 provide HAC-FP deployment with sink nodes in which higher transmission energy is dissipated to nearby nodes which minimizes power utilization of nodes.

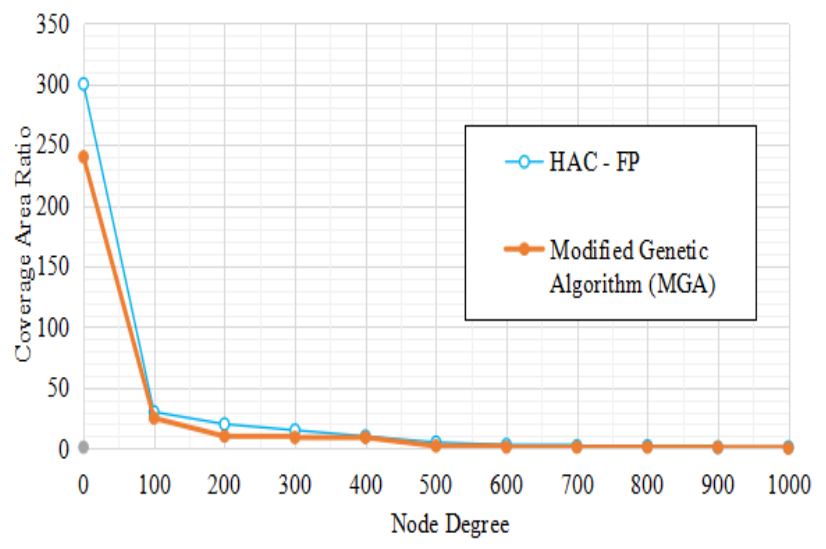

Fig.9. The ratio of the coverage area for HAC-FP.

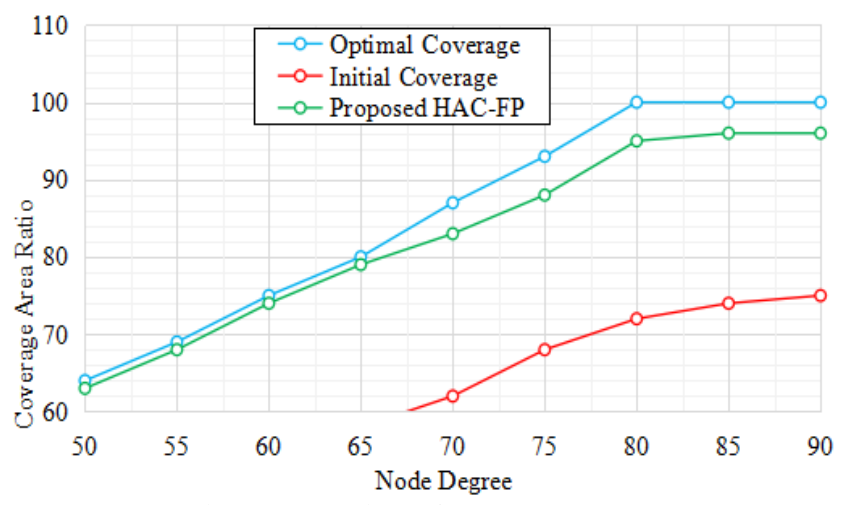

Fig.10. Comparison of HAC-FP coverage.

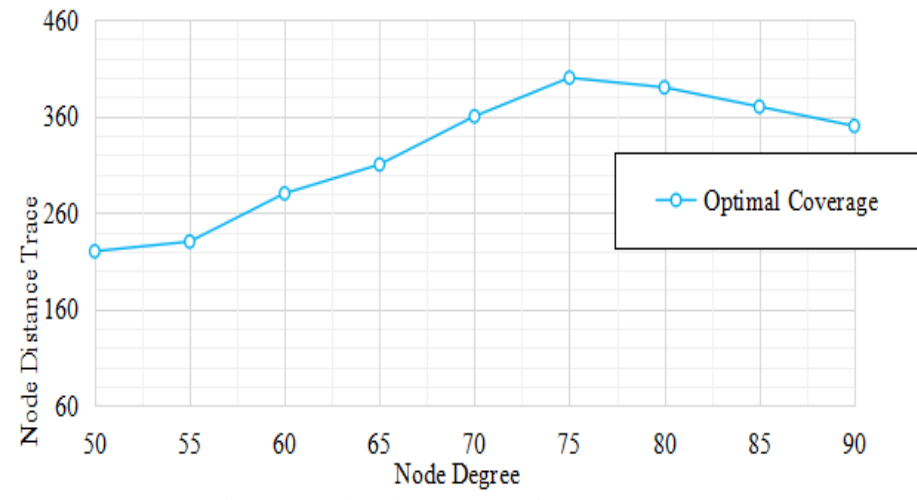

Fig. 11. Optimal Coverage of HAC-FP.

In the above figure, nodes are implied with respect to exponential time in which the proposed approach HAC-FP performs effectively for dynamic routing with minimal collision.

\section{CONCLUSION}

Mobile Ad-hoc Network belongs to the category of ad-hoc network with sensing devices with minimal cost and low energy conservation with interconnected wireless links. Another advantage of a Mobile Ad-hoc Network is limited resource constraints and compact sensor nodes. The proposed HAC-FP makes use of neighborhood distance to determine the best position for sensor nodes. With hypersphere localization, the Levy struggle in flower pollination is used to find the best energy position. The first phase, which is based on hyperspheres, is used to locate sensor nodes. Sensor network node energy consumption is lowered based on neighborhood distance. Matlab Simulator was used to test the performance of HAC-FP, and the findings showed that the suggested method increased throughput while consuming less energy.

\section{REFERENCES}

[1] Akyildiz I. F., Su W., Sankarasubramaniam Y., \& Cayirci E. Mobile Ad-hoc Networks: a survey.Computer networks, 2002;38(4):393-422.

[2] Sivakumar S., \& Venkatesan R. Meta-heuristic approaches for minimizing error in localization of Mobile Ad-hoc Networks. Applied soft computing, 2015; 36:506-518.

[3] Bulusu N., Heidemann J., Estrin D., \& Tran T. Self-configuring localization systems: Design and experimental evaluation. ACM Transactions on Embedded Computing Systems (TECS), 2004;3(1), 2460.

[4] Gungor V. C., \& Hancke G. P. Industrial Mobile Ad-hoc Networks: Challenges, design principles, and technical approaches. IEEE Transactions on industrial electronics, 2009;56(10):4258-4265.

[5] Shiu Y. S., Chang S. Y., Wu H. C., Huang S. C. H., \& Chen H. H. Physical layer security in wireless networks: A tutorial. IEEE Wireless Communications, 2011;18(2).

[6] Nardelli P. H., Alves H., De Lima C. H., \& Latva-Aho M. Throughput maximization in multi-hop wireless networks under a secrecy constraint. Computer Networks, 2016;109:13-20.

[7] Moshizi M. M., Bardsiri V. K., \& Heydarabadipour E. The Application of Meta-Heuristic based Clustering Techniques in Mobile Ad-hoc Networks. International Journal of Control and Automation, 2015; 8(3):319-328.

[8] Branch B., \& Bardsir I. The Application of Meta-Heuristic based Clustering Techniques in Mobile Ad-hoc Networks. International Journal of Control and Automation, 2015; 8(3):319-328.

[9] Prasad D. R., Naganjaneyulu P. V., \& Prasad K. S. Metaheuristic techniques for cluster selection in MANET. In Algorithms, Methodology, Models, and Applications in Emerging Technologies (ICAMMAET), 2017 International Conference on, pp. 1-6. IEEE.

[10] Zeng Z., Yu X., He K., Huang W., \& Fu Z. Iterated tabu search and variable neighborhood descent for packing unequal circles into a 
circular container. European Journal of Operational Research, 2016;250(2):615-627.

[11] Adickes M. D., Billo R. E., Norman B. A., Banerjee S., Nnaji B. O., \& Rajgopal J. A. Optimization of indoor wireless communication network layouts. Iie Transactions, 2002;34(9):823-836.

[12] Birgin E. G., Martinez J. M., \&Ronconi D. P. Optimizing the packing of cylinders into a rectangular container: a nonlinear approach. European Journal of Operational Research, 2005;160(1):19-33.

[13] Castillo I., Kampas F. J., \&Pintér J. D. Solving circle packing problems by global optimization: numerical results and industrial applications. European Journal of Operational Research, 2008;191(3):786-802.

[14] Tahir N. H. M., \&Atan F. A Modified Genetic Algorithm Method for Maximum Coverage in Dynamic Mobile Mobile Ad-hoc Networks. Journal of Basic and Applied Scientific Research, 2016; Basic. Appl. Sci. Res., 6(11)26-32.

[15] Baccelli F., El Gamal A., \& David N. C. Interference networks with point-to-point codes. IEEE Transactions on Information Theory, 2011;57(5):2582-2596.

[16] Gomez-Cuba F., Asorey-Cacheda R., \& Gonzalez-Castano F. J. A survey on cooperative diversity for wireless networks. IEEE Communications Surveys \& Tutorials, 2012;14(3):822-835.

[17] Haenggi M. Stochastic geometry for wireless networks. Cambridge University Press. 2012; Cambridge University Press, 9781139043816.

[18] Inaltekin H., Chiang M., Poor H. V., \& Wicker S. B. On unbounded path-loss models: effects of singularity on wireless network performance. IEEE Journal on Selected Areas in Communications, 2009;27(7).

[19] Nardelli P. H., Kountouris M., Cardieri P., \& Latva-Aho M. Throughput optimization in wireless networks under stability and packet loss constraints. IEEE Transactions on Mobile Computing, 2014;13(8):1883-1895.

[20] Benaya A. M., Shokair M., El-Rabaie E. S., \&Elkordy M. F. Optimal power allocation for sensing-based spectrum sharing in MIMO cognitive relay networks. Wireless Personal Communications, 2015; 82(4):2695-2707.

[21] Rosas-Casals M., Valverde S., \& Solé R. V. Topological vulnerability of the European power grid under errors and attacks. International Journal of Bifurcation and Chaos, 2007;17(07):2465-2475.

[22] Panaitopol D., Datta R., \& Fettweis G. Cyclostationary detection of cognitive radio systems using GFDM modulation. In 2012 IEEE Wireless Communications and Networking Conference (WCNC), pp. 930-934. IEEE.

[23] Inzucchi S. E., Bergenstal R. M., Buse J. B., Diamant M., Ferrannini E., Nauck M., \& Matthews D. R. Management of hyperglycemia in type 2 diabetes, 2015: a patient-centered approach: update to a position statement of the American Diabetes Association and the European Association for the Study of Diabetes. Diabetes care, 2015;38(1):140149.

[24] Zewail I., Saad W., Shokair M., \&El_dolil S. A. Maximizing the total throughput for GFDM system using hybrid PSO-PS algorithm. Journal of Electrical Systems and Information Technology, 2017.

[25] Lin H., \& Siohan P. Orthogonality improved GFDM with low complexity implementation. In 2015 IEEE Wireless Communications and Networking Conference (WCNC), pp. 597-602. IEEE. 